\title{
Low Energy Cross-Sections for Positron Interactions with Cyclic Hydrocarbons
}

\author{
G.P. Karwasz ${ }^{a}$, D. Pliszka ${ }^{b}$, R.S. Brusa ${ }^{a}$ And C. Perazzolli ${ }^{a}$ \\ ${ }^{a}$ Dipartimento di Fisica, Università di Trento, 38050 Povo (TN), Italy \\ ${ }^{b}$ Institute of Physics, Pomeranian Pedagogical Academy, 76-200 Słupsk, Poland
}

We report total cross-section measurements for positron scattering on cyclic hydrocarbons: benzene, aniline, and cyclohexane. Measurements were done by an absolute transition method in the energy range $1.5-20 \mathrm{eV}$ (0.4-20 eV for cyclohexane). High cross-sections for all measured molecules were observed at low energies. In the case of cyclohexane the cross-section in the zero energy limit tends to a constant value. In aniline a weakly accented peak slightly above the positronium formation threshold is observed. Similar but less visible bump was observed in the case of benzene. Measurement of total cross-sections in nitrogen and argon used for calibration of the apparatus are also presented.

PACS numbers: $34.85 .+\mathrm{x}, 39.90 .+\mathrm{d}$

\section{Introduction}

Positron scattering on molecules and atoms in gas phase is under research from early seventies. The interaction between positron and molecules is weaker than in the case of electron scattering, due to a partial compensation of the attractive polarization potential by the static potential which is repulsive for positrons; additionally, the exchange potential is absent for positrons. Therefore, scattering of positrons compared to electrons is a complementary tool in studying atomic and molecular structure. Experimental difficulties arise from low intensities of positron beams, typically at least three orders of magnitude lower than electron beams. Measurements of total cross-sections in the low energy range were performed for several linear hydrocarbons by Sueoka's group [1-3] and for methane by Kauppila and Stein's group [4]. Numerous measurements exists also for noble gases [4-6]. The total cross-section can be measured precisely by an absolute method from the beam attenuation. On the other hand, it can be considered as a sum of partial cross-sections for elastic and numerous inelastic processes, like vibrational, electronic excitations, ionization, positronium formation. These cross-sections are needed for modeling positron slowing down in matter and, indirectly, for positron annihilation rates, but are difficult to be measured by absolute methods. 
The annihilation rates in the gas phase for energy-selected positrons have been studied starting from nineties by Surko and collaborators [7]. They noticed for a number of targets, including Ar and hydrocarbons, that at low energies an effective atomic number for positron annihilation $Z_{\text {eff }}$ rises sharply towards the zero scattering energy. For example, the effective atomic number for $\mathrm{C}_{4} \mathrm{H}_{10}$ amounts to $Z_{\text {eff }}=10$ at $2 \mathrm{eV}$ scattering energy and to as much as $Z_{\text {eff }}=20,000$ in the meV range. In more recent measurements $[8,9]$ performed with the energy resolution as good as $25 \mathrm{meV}$ Surko and collaborators observed for a number of hydrocarbons a sharp enhancement of $Z_{\text {eff }}$ slightly below thresholds for molecular vibrations, indicating a kind of resonant process.

A new apparatus has been constructed for total cross-section measurements at low energies $(<20 \mathrm{eV})$ in gas phase at Trento University [10]. Here, we present first results for three hydrocarbons, nitrogen, and argon.

\section{Apparatus}

The design of the beam and the detailed description of the apparatus have been presented elsewhere [10]. An electrostatic guiding is used in the first part of optics and a rather weak (10 Gs) magnetic field with electrostatic focusing in the second part. Present setup differs from Surko's apparatus, who uses high intensity (kGs) guiding magnetic fields.

Dimensions of the scattering cell were $1 \mathrm{~mm}$ diameter of entrance and exit apertures, and $10 \mathrm{~cm}$ in length. The angular resolution of the detector was equal to $3.1 \times 10^{-4} \mathrm{sr}$; this is two orders of magnitude better than in the apparatus of Sueoka and collaborators $\left(3.2 \times 10^{-2} \mathrm{sr}\right)$. A worse angular resolution usually leads to underestimation of cross-sections.

As a positron source ${ }^{22} \mathrm{Na}$ isotope of $15 \mathrm{mCi}$ activity was used. In front of the source a $1 \mu \mathrm{m}$ thick tungsten monocrystal moderator from Aarhus University [11] was placed. It was annealed at more than $2000^{\circ} \mathrm{C}$ in situ in UHV $\left(10^{-8} \mathrm{Tr}\right)$ conditions.

The apparatus was originally designed [12] in a remoderator configuration [13]: positrons with $2-5 \mathrm{keV}$ energy were to be focused to a spot of $0.1 \mathrm{~mm}$ diameter, injected into a copper film, reemitted and only then guided to the scattering cell. The focusing, the energy loss, and the low mean energy of reemitted positrons (less than $1 \mathrm{eV}$ from $\mathrm{Cu}$ compared to $2.3 \mathrm{eV}$ from $\mathrm{W}$ [14]) would result in a beam brightness enhancement. However, the preparation of the copper film of good quality is not a trivial task and at present stage the apparatus was used in a decelerating mode. Positrons instead of being accelerated to a remoderator were directly decelerated down to $1-20 \mathrm{eV}$ before entering the scattering cell. Due to a worse angular spread of the beam in this mode, the positron beam intensity falls down from about $4000 \mathrm{e}^{+} / \mathrm{s}$ before deceleration to $100 \mathrm{e}^{+} / \mathrm{s}$ and $10 \mathrm{e}^{+} / \mathrm{s}$ inside the scattering cell at $10 \mathrm{eV}$ and $1 \mathrm{eV}$ collision energy, respectively. 
A glass-based channeltron is used as a detector. The separating $90^{\circ}$ bent between the first and second optical columns [10] and good shielding of the source area allow to obtain extremely low background level on the channeltron, less than $0.05 \mathrm{e}^{+} / \mathrm{s}$.

Due to the lack of any known sharp structure in total cross-sections for positron scattering, the energy calibration of the apparatus was obtained from the positronium formation threshold (i.e. $8.8 \mathrm{eV}$ in $\mathrm{N}_{2}$ and $8.96 \mathrm{eV}$ in $\mathrm{Ar}$, as calculated from literature ionization energies), where the cross-section shows a steep rise, see Fig. 1. The estimated shift amounts to $+2.4 \pm 0.1 \mathrm{eV}$ and is close to the literature work function for positrons in tungsten [15].

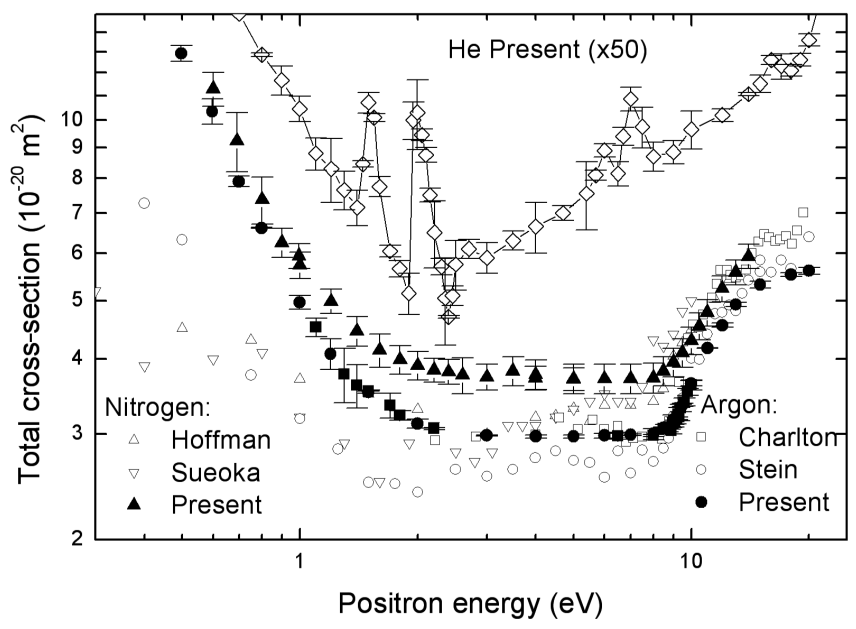

Fig. 1. Comparison between present data for: $\mathrm{N}_{2}$ (full triangles) and those by Hoffman et al. [16], Sueoka and Hamada [3]; Ar (full circles) and those by Charlton et al. [5] and Kauppila et al. [17] — determination of the energy scale.

In absolute method, the gas pressure, its temperature and the positron beam intensity with $(I)$ and without $\left(I_{0}\right)$ gas in the scattering cell are monitored. The cross-section $\sigma$ is obtained from de Beer-Lambert's attenuation formula

$$
I=I_{0} \exp (-p l \sigma / k T),
$$

where $l$ is the length of the scattering cell, $p$ is the gas pressure, $T$ is temperature of the gas and $k$ is Boltzmann's constant. The pressure was determined by a capacitance meter Leybold Inficon CR091 with better than 1\% precision. The pressure readout precision is the main source of possible systematic errors in our data. The measurements were performed in long series of constant pressure, changing the positron energy. The experimental points shown in Figs. 1-3 are mean values of 6-10 runs, each performed as 20 values of $10 \mathrm{~s}$ count accumulation with gas on and off. 


\section{Results}

Our data both for nitrogen and argon show a constant cross-section above a few eV up to the positronium formation threshold, see Fig. 1. For $\mathrm{N}_{2}$ we use the data of Hoffman et al. [16] and the data from Sueoka's group [3] for comparison. In the case of Ar our data are compared to those of Charlton et al. [5] and those from the Detroit laboratory [17]. Present $\mathrm{N}_{2}$ data are in a good agreement with measurements of Hoffman et al. [16] above the positronium formation threshold but at lower energies are higher by about $13 \%$. For Ar a good agreement between present results and Kauppila et al.'s [17] data above the positronium formation threshold is observed. In the low energy range our total cross-sections are generally higher than those from other measurements. This can arise from a better angular resolution of present apparatus as compared to previous experiments.

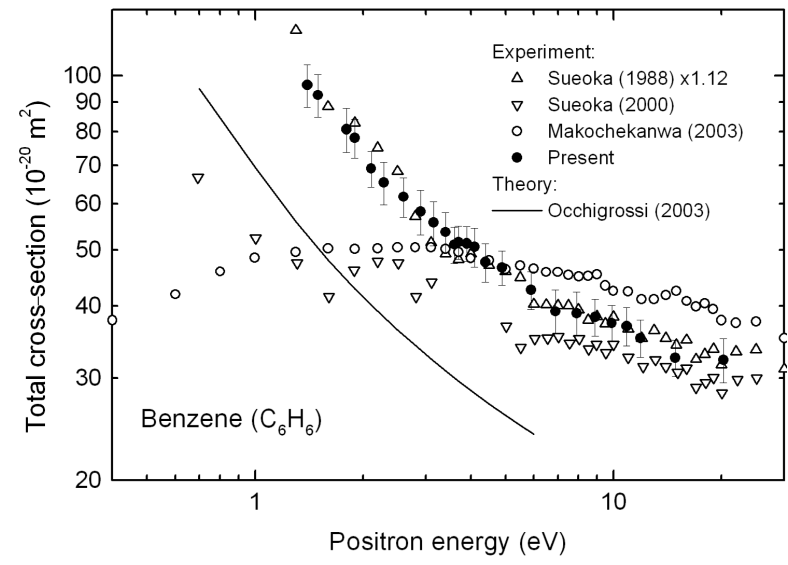

Fig. 2. Total cross-sections for positron scattering on benzene. Full circles - present measurements, triangles - data of Sueoka [2] normalized by $+12 \%$; open circles and inverted triangles - data of Sueoka and collaborators [1, 21], respectively, corrected by them for the angular distribution of scattered positrons. Line - theory of Occhigrossi and Gianturco [23].

In Fig. 2 we present data in benzene compared to the first [2] and the most recent [1] measurements of Sueoka and co-workers. We note that present data are in a very good agreement with the first measurements but after their normalization by $+12 \%$. The needed normalization is due to using an "effective" length of the scattering cell by Sueoka. Such an underestimation of the total cross-section was also noted [18] in electron cross-sections of the Sueoka group: in methane the data of Sueoka and Mori [19] are about 10\% lower than data of other groups.

Newer cross-sections of Sueoka's group are corrected [1, 20, 21] due to the angular resolution of their apparatus. This procedure, useful for electrons, can be risky for positrons where angular distributions of scattered particles are not 
known. This is the probable reason that present data do not agree in values and in shape with the newer measurement of Sueoka and collaborators [1, 21], see Fig. 2. In particular we do not observe a broad maximum at about $2 \mathrm{eV}$ (see inverted triangles in Fig. 2) as reported by Kimura et al. [20] and Sueoka et al. [21]. Instead, we see a small, bump-like structure, outside the statistical error bar, slightly above the threshold for positronium formation. Subtracting a monotonically varying background, we obtain a value of about $5 \times 10^{-20} \mathrm{~m}^{2}$ for the height of this structure. This value coincides with the evaluation of the positronium-formation cross-sections, $6 \times 10^{-20} \mathrm{~m}^{2}$, done by Sueoka [2] in his total cross-section measurements and does not with the positronium formation in hydrogen [22].

Very high values of the cross-section in benzene in the low energy range (about $100 \AA^{2}$ at $1.5 \mathrm{eV}$ ) are quite surprising. Such a rise at low energies is predicted by the theory of Occhigrossi and Gianturco [23] but their absolute values are lower by a factor of two. High values of the elastic cross-section in benzene in the low energy limit have been attributed [23] to the high value of the dipole polarizability $\left(69.6 a_{0}^{3}\right)$.

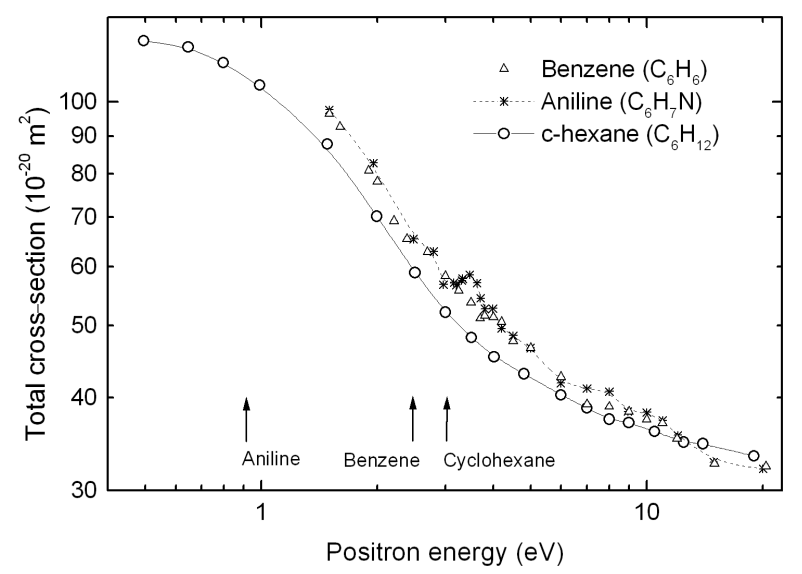

Fig. 3. Comparison between present data for benzene, aniline and cyclohexane (line is added only for an eye-guide reasons). Arrows indicate positronium formation thresholds.

In order to check if this high total cross-section is due to trapping of positron inside the benzene ring, the total cross-section in aniline $\left(\mathrm{C}_{6} \mathrm{H}_{5} \mathrm{NH}_{2}\right)$ has been measured. We expected smaller cross-section values, due to the effect of localization of hybridized $s p^{2}$ electrons in aniline. Surprisingly, the cross-section in two targets are identical, within our experimental error bar, in the entire 1-20 eV energy range (Fig. 3). The functional group $\mathrm{NH}_{2}$ present in aniline does not change much the cross-section, in spite of a higher charge density inside the ring in benzene. The only noticeable difference is again the bump above threshold for positronium formation (2.4 eV in benzene, $0.9 \mathrm{eV}$ in aniline), slightly visible in the case of 
benzene and more accented in aniline (see Fig. 3). In cyclohexane, similarly to benzene and aniline, a high cross-section $\left(120 \AA^{2}\right.$ at $\left.0.5 \mathrm{eV}\right)$ has been obtained. However, up to $5 \mathrm{eV}$, the cyclohexane cross-section is lower by $8 \%$ than that in benzene, converging to it at higher energies. The cross-section in cyclohexane shows no visible bumps; in the zero energy limit it tends to a constant value of about $150 \times 10^{-20} \mathrm{~m}^{2}$.

\section{Cross-sections and annihilation rates}

Comparison between present total cross-sections and annihilation rates in both gas and liquid phase is not straightforward. Surko et al. [24] in the gas phase measured the effective atomic number of 185,000 for cyclohexane and 18,400 for benzene in spite of the higher total cross-section for the latter target, as presently measured. This would suggest that high values of total cross-sections are due to elastic scattering, as by the way indicated by the theory [23], and not due to resonant processes. Resonant processes can, instead, influence trapping of positrons and subsequently cause high annihilation rates.

TABLE

Lifetime parameters of the liquids, $\tau_{1}$ - annihilation of para-positronium, $\tau_{2}$ - lifetime of free positrons, $\tau_{3}$ - lifetime of ortho-positronium, from Ref. [26].

\begin{tabular}{c|c|c|c|c|c|c|c}
\hline \hline Molecule & Formula & $T_{1}[\mathrm{~ns}]$ & $I_{1}[\%]$ & $\tau_{2}[\mathrm{~ns}]$ & $I_{2}[\%]$ & $\tau_{3}[\mathrm{~ns}]$ & $I_{3}[\%]$ \\
\hline benzene & $\mathrm{C}_{6} \mathrm{H}_{6}$ & 0.142 & 20.5 & 0.440 & 36.4 & 3.18 & 43.1 \\
cyclohexane & $c-\mathrm{C}_{6} \mathrm{H}_{12}$ & 0.214 & 26.1 & 0.469 & 36.3 & 3.25 & 37.6 \\
aniline & $\mathrm{C}_{6} \mathrm{H}_{7} N_{2}$ & 0.204 & 38.4 & 0.477 & 28.6 & 2.37 & 32.9
\end{tabular}

In liquids, in general, positron lifetimes depend on cross-sections, but detailed analysis requires knowledge of partial cross-sections down to zero energy. To our knowledge, such an analysis was done in He and Ar solely [25]. We note, see Table, for the three hydrocarbons studied here similar values of $\tau_{2}$ lifetime component (i.e. annihilation in flight) and a high probability of para-positronium formation in aniline (38.4\% intensity of the shortest lifetime component [26]). We recall a more prominent positron formation bump in the cross-section for aniline than in benzene. Chemical effects, as on the other hand noticed already in early measurements [27] seem to play a more important role in annihilation in liquids than cross-sections themselves. Further measurements, possibly also of partial cross-sections and in a wide energy range would be desirable.

\section{Acknowledgments}

We acknowledge support from EPIC ("Electron and Positron Induced Chemistry") European Union Training for Mobility and Research and EIPAM ("Electron Induced Processes in Molecules") European Science Foundation projects. 


\section{References}

[1] C. Makochekanwa, O. Sueoka, M. Kimura, Phys. Rev. A 68, 32707 (2003).

[2] O. Sueoka, J. Phys. B, At. Mol. Phys. 21, L631 (1988).

[3] O. Sueoka, A. Hamada, J. Phys. Soc. Jap. 62, 2669 (1993).

[4] W.E. Kauppila, T.S. Stein, Adv. Phys. 26, 1 (1990).

[5] M. Charlton, G. Laricchia, T.C. Griffith, G.L. Wright, G.R. Heyland, J. Phys. B 17, 4945 (1984).

[6] B. Jaduszliwer, D.A.L. Paul, Can. J. Phys. 51, 1565 (1973); 52, 1047 (1974).

[7] T.J. Murphy, C.M. Surko, Phys. Rev. A 46, 5696 (1992).

[8] K. Iwata, G.F. Gribakin, R.G. Greaves, C. Kurz, C.M. Surko, Phys. Rev A 61, 22719 (2000).

[9] S.J. Gilbert, L.D. Barnes, J.P. Sullivan, C.M. Surko, Phys. Rev. Lett. 88, 043201 (2002).

[10] G.P. Karwasz, R.S. Brusa, M. Barozzi, A. Zecca, Nucl. Instrum. Methods Phys. Res. B 171, 178 (2000).

[11] N. Zafar, J. Chevalier, F.M. Jacobson, M. Charlton, G. Laricchia, Appl. Phys. A 47, 409 (1988).

[12] A. Zecca, R.S. Brusa, M. Bettonte, E. Rajch, S. Mariazzi, G.P. Karwasz, Radiat. Phys. Chem. J. 68, 319 (2003).

[13] A.P. Mills Jr, Appl. Phys. 23, 189 (1980).

[14] D.A. Fischer, K.G. Lynn, D.W. Gidley, Phys. Rev. B 33, 4479 (1986).

[15] P. Van Reeth, J.W. Humberston, Nucl. Instrum. Methods Phys. Res. B 171, 106 (2000).

[16] K.R. Hoffman, M.S. Dababneh, Y.-F. Hsieh, W.E. Kauppila, V. Pol, J.H. Smart, T.S. Stein, Phys. Rev. A 25, 1393 (1982).

[17] W.E. Kauppila, T.S. Stein, G. Jesion, Phys. Rev. Lett. 36, 580 (1976).

[18] G.P. Karwasz, A. Zecca, R.S. Brusa, La Rivista del Nuovo Cimento 24, 1 (2001).

[19] O. Sueoka, S. Mori, J. Phys. B, At. Mol. Phys. 19, 4035 (1986).

[20] M. Kimura, O. Sueoka, A. Hamada, Y. Itikawa, Adv. Chem. Phys. 111, 537 (1999).

[21] O. Sueoka, M.K. Kawada, M. Kimura, Nucl. Instrum. Methods Phys. Res. B 171, 96 (2000).

[22] S. Zhou, H. Li, W.E. Kauppila, C.K. Kwan, T.S. Stein, Phys. Rev. A 55, 361 (1997).

[23] A. Occhigrossi, F.A. Gianturco, J. Phys. B, At. Mol. Phys. 36, 1383 (2003).

[24] T.J. Murphy, C.M. Surko, Phys. Rev. Lett. 67, 2954 (1991).

[25] I. Pepe, Paul Dal, J. Steyaert, F. Gimenonogues, J. Deutsch, R. Prieels, J. Phys. B 28, 3643 (1995).

[26] O.E. Mogensen, Positron Annihilation in Chemistry, Springer-Verlag, Berlin 1995.

[27] P.R. Gray, C.F. Cook, G.P. Sturm Jr, J. Chem. Phys. 48, 1145 (1967). 